\title{
Interleukin-17A Levels Increase in Serum of Children With Juvenile Idiopathic Arthritis
}

\author{
Gordana VIJATOV-DJURIC, ${ }^{1,2}$ Aleksandra DORONJSKI, ${ }^{1,2}$ Igor MITIC, $, 1,3$ Snezana BRKIC,,${ }^{1,4}$ Nenad BARISIC ${ }^{1,2}$ \\ ${ }^{1}$ Faculty of Medicine, University of Novi Sad, Novi Sad, Serbia \\ ${ }^{2}$ Department of Pediatrics, Institute for Child and Youth Health Care of Vojvodina, Novi Sad, Serbia \\ ${ }^{3}$ Department of Immunology and Nephrology, Clinical Centre of Vojvodina, Novi Sad, Serbia \\ ${ }^{4}$ Clinic for Infectious Diseases, Clinical Centre of Vojvodina, Novi Sad, Serbia
}

\begin{abstract}
Objectives: This study aims to determine the serum levels of interleukin-17A (IL-17A) in children with juvenile idiopathic arthritis (JIA) and analyze the correlation between IL-17A values and disease activity, certain clinical features, and laboratory markers of inflammation.

Patients and methods: The study included 30 children ( 7 boys, 23 girls; mean age $8.8 \pm 5.3$ years; range 1 to 18 years), who had been diagnosed with JIA (18 children were diagnosed during the study period and 12 children were diagnosed before the start of the study) and had active disease during the study period. Control group included 30 healthy, age- and sex- matched children (9 boys, 21 girls; mean age $8.3 \pm 4.8$ years; range 1 to 18 years). The enzyme-linked immunosorbent assay was used to assess the serum IL-17A levels of children with JIA in the active phase of the disease and control group. Clinical and laboratory features of the disease were evaluated for the children with JIA.

Results: Serum levels of IL-17A in children with JIA were significantly higher in comparison to control group. In children with JIA who were prospectively monitored, statistically significantly decreased IL-17A level was recorded in the inactive phase of the disease. The incidence of arthritis of coxofemoral joints was significantly more common, and the mean levels of erythrocyte sedimentation rate and C-reactive protein were significantly higher in the group of children with JIA with detectable levels of IL-17A. Children with JIA and detectable levels of IL-17A had significantly higher values of Juvenile Arthritis Disease Activity Score-27 in comparison to children with JIA and non-detectable IL-17A.

Conclusion: Assessment of serum IL-17A levels in early phases of JIA gives an opportunity for early detection of children that have higher risk for worse functional outcome.

Keywords: Disease activity; interleukin-17A; juvenile idiopathic arthritis.
\end{abstract}

Over the past several years, a number of studies have been conducted which point to the role of interleukin-17A (IL-17A) in the development of chronic arthritis. IL-17A has a role both at the initial stages of joint inflammation and in the destruction of joint cartilage and bone structures. ${ }^{1}$ IL-17A binds to its receptors -IL-17 receptor A/IL-17 receptor C- on synoviocytes, endothelial cells, fibroblasts and osteoblasts and stimulates production of pro-inflammatory cytokines, chemokines and other inflammatory mediators, and also, interacts synergistically with other proinflammatory cytokines such as IL-1, IL-6, and tumor necrosis factor alpha. . $^{2,3}$
In the initial phases of chronic arthritis, IL-17A affects synovial hypercellularity. ${ }^{4}$ A study conducted on the culture of synovial fibroblasts has shown that IL-17A stimulates the production of the vascular endothelial growth factor, which is important in the angiogenesis and the consequent synovial proliferation. ${ }^{5}$ In addition, IL-17A stimulates increased synoviolin expression, an E3 ubiquitin ligase, which causes an imbalance between proliferation and apoptosis of synoviocytes. ${ }^{6}$

Interleukin-17A stimulates the release of degradation enzymes from the matrix metalloproteinases family, which cause cartilage

Received: May 15, 2016 Accepted: October 10, 2016 Published online: April 04, 2017

Correspondence: Nenad Barisic, MD. Department of Immunology and Nephrology, Clinical Centre of Vojvodina, 21000 Novi Sad, Serbia.

Tel: +381216366540 e-mail:nenad.barisic@mf.uns.ac.rs

(2017 Turkish League Against Rheumatism. All rights reserved. 
destruction. The members of this family take part in the degradation of the extracellular matrix of the cartilage, primarily of proteoglycan and collagen. ${ }^{7,8}$ IL-17A may inhibit the synthesis of cartilage matrix proteins by direct influence on the chondrocytes. ${ }^{7}$ One of the crucial roles of IL-17A is the activation and mobilization of neutrophils. The increase in the neutrophil influx to the joints and the release of their proteolytic enzymes result in the degradation of the cartilage matrix and destruction of chondrocytes. ${ }^{9}$

The ultimate effect of IL-17A activity on the bone structure is the destruction of the bone. The research into cell cultures in animal models identified various mechanisms of IL-17A activity, which in sum make it a destructive cytokine locally. ${ }^{10,11}$ One of the effects of IL-17A on the bone structure is the disturbance of the receptor activator relationship between nuclear factor kappa beta ligand and osteoprotegerin. On the one hand, IL-17A increases the expression of receptor activator of nuclear factor kappa beta ligand, which has a central role in the stimulation of osteoclastogenesis, and on the other, it inhibits the expression of osteoprotegerin, which reduces the production of osteoclasts. ${ }^{12-14}$ Also, IL-17A enhance expression of macrophage-colony stimulating factor which has an important role in the differentiation and sustention of osteoclasts. ${ }^{2}$ The negative correlation of osteoblastogenesis and the IL-17A level in the synovial fluid in children with juvenile idiopathic arthritis (JIA) confirms the role of IL-17A in the lesion in bone structures. ${ }^{15}$ The destruction of bone structures is also influenced by various proinflammatory mediators induced by IL-17A, such as IL-6, prostaglandin E2, nitric oxide, and others. ${ }^{10,16}$

At juvenile age, chronic joint inflammation is primarily manifested as JIA. The main criteria for diagnosing JIA are disease onset under the age of 16 , arthritis of one or more joints, and continuous duration of arthritis for at least six weeks, where all other rheumatic and non-rheumatic diseases are excluded. ${ }^{17}$ The International League of Associations for Rheumatology classification system (second revision) divides JIA into seven subtypes: systemic arthritis, oligoarthritis, rheumatoid factor (RF) negative polyarthritis, RF positive polyarthritis, enthesitis-related arthritis, psoriatic arthritis, and undifferentiated arthritis. ${ }^{18}$
Cytokines have a crucial role in maintaining the inflammatory process in children with JIA. The balance of cytokine secretion is disturbed, so the proinflammatory profile of cytokine is predominant, and their elevated level, both in peripheral blood and/or in synovial fluid, correlates with the activity of the disease. ${ }^{19,20}$ To our knowledge, only a limited number of studies have been carried out about the role of IL-17A in the pathogenesis of JIA. Most of those studies concluded that levels of IL-17A in synovial fluid and synovial tissue are increased in children with JIA. ${ }^{21-23}$ Stimulation of synovial fibroblasts of patients with JIA using recombinant IL-17A causes a multi-fold increase of IL-6, matrix metalloproteinase- 1 and matrix metalloproteinase- $3 .^{22}$ The results of these studies pinpoint possible inflammatory and destructive potential of IL-17A in the pathogenesis of chronic arthritis in children.

In this study, we aimed to determine the serum levels of IL-17A in children with JIA and analyze the correlation between IL-17A values and disease activity, certain clinical features, and laboratory markers of inflammation.

\section{PATIENTS AND METHODS}

The study, which was conducted at regional University hospital (Institute for Child and Youth Health Care of Vojvodina) between January 2011 and December 2013, included 30 children (7 boys, 23 girls; mean age $8.8 \pm 5.3$ years; range 1 to 18 years), who had been diagnosed with JIA (18 children were diagnosed during the study period and 12 children were diagnosed before the start of the study) and had active disease during the study period. Control group included 30 healthy, age- and sex- matched children ( 9 boys, 21 girls; mean age $8.3 \pm 4.8$ years; range 1 to 18 years).

The study protocol was approved by the Hospital Ethics Committee. A written informed consent was obtained from all parents and children over the age of 10 . The study was conducted in accordance with the principles of the Declaration of Helsinki.

Diagnosis and classification of JIA were established according to International League of Associations for Rheumatology revised criteria 


\begin{tabular}{|c|c|c|c|c|c|}
\hline \multirow[t]{2}{*}{ Clinical parameters } & \multicolumn{5}{|c|}{ Juvenile idiopathic arthritis patients $(n=30)$} \\
\hline & $\mathrm{n}$ & $\%$ & Mean \pm SD & Mean & Range \\
\hline \multicolumn{6}{|l|}{ Sex } \\
\hline Female & 23 & 77 & & & \\
\hline Male & 7 & 23 & & & \\
\hline Age at disease onset (year) & & & $6.5 \pm 5.2$ & & \\
\hline Age at study visit (year) & & & $8.8 \pm 5.3$ & & \\
\hline Duration of the disease (year) & & & $2.3 \pm 3.4$ & & \\
\hline \multicolumn{6}{|l|}{ JIA subtype } \\
\hline Oligoarthritis & 17 & 57 & & & \\
\hline Rheumatoid factor negative polyarthritis & 7 & 23 & & & \\
\hline Rheumatoid factor positive polyarthritis & 1 & 3 & & & \\
\hline Systemic arthritis & 2 & 7 & & & \\
\hline Enthesitis-related arthritis & 2 & 7 & & & \\
\hline Psoriatic arthritis & 1 & 3 & & & \\
\hline \multicolumn{6}{|l|}{ Disease activity } \\
\hline Juvenile Arthritis Disease Activity Score-27 & & & & 15 & $6-47$ \\
\hline \multicolumn{6}{|l|}{ Therapy at study visit* } \\
\hline Nonsteroidal anti-inflammatory drugs & 25 & 83 & & & \\
\hline Methotrexate & 9 & 30 & & & \\
\hline Sulfasalazine & 1 & 3 & & & \\
\hline Etanercept & 1 & 3 & & & \\
\hline Systemic corticosteroids & 5 & 17 & & & \\
\hline Intra-articular corticosteroids & 5 & 17 & & & \\
\hline
\end{tabular}

for JIA. ${ }^{18}$ Clinical characteristics of patients with JIA are shown in Table 1. Activity of JIA was established based on the Juvenile Arthritis Disease Activity Score-27 (JADAS-27). ${ }^{24}$ The following information were collected: age at onset of JIA, duration of disease, number and localization of joint with active arthritis (joint with active arthritis is a joint with swelling not due to bony enlargement or, if no swelling is present, limitation of motion accompanied by either pain on motion and/or tenderness (The American College of Rheumatology definition), extra-articular clinical manifestations of JIA (enthesitis, acute uveitis, intermittent fever that lasts two or more weeks, transient erythematous rash, serositis, splenomegaly and generalized lymphadenopathy), erythrocyte sedimentation rate (ESR), C-reactive protein (CRP), leukocyte, hemoglobin, and thrombocyte counts. Exclusion criteria were acute infections in the last four weeks, chronic infection, other concurrent chronic diseases, immunodeficiency or orthopedic diseases.

Serum levels of IL-17A were determined in all 60 participants. Also, eight children with JIA (six with oligoarthritis and two with RF negative poliarthritis) were followed-up prospectively. The values of IL-17A levels in these eight patients were determined during the active phase of the disease and then again during the inactive phase (when Wallace criteria for clinically non-active phase of the disease were fulfilled and when JADAS-27 was less than 1). ${ }^{25,26}$

The disease was considered to be inactive when there were no joints with active arthritis; no fever, rash, serositis, splenomegaly, or generalized lymphadenopathy attributable to JIA; no active uveitis as defined by the Standardization of Uveitis Nomenclature Working Group; when ESR and CRP levels were within normal limits, or if elevated, not attributable to JIA; when best possible physician's global assessment of disease activity score on the scale was used, and when duration of morning stiffness was $\leq 15$ minutes according to Wallace criteria. ${ }^{25}$

Measurement of IL-17A in the serum was carried out in the laboratory of the Clinical Centre of Vojvodina in Novi Sad. The levels of IL-17A were determined by the quantitative enzymelinked immunosorbent assay, which contains the recombinant human IL-17A and biotinilized antibody targeting human IL-17A (RayBio ${ }^{\circledR}$ Human 


\begin{tabular}{|c|c|c|c|c|}
\hline \multirow{3}{*}{ Group } & \multicolumn{4}{|c|}{ Number of children (\%) } \\
\hline & \multicolumn{2}{|c|}{ IL-17A $\geq 15.63 \mathrm{pg} / \mathrm{mL}$} & \multicolumn{2}{|c|}{ IL-17A $<15.63 \mathrm{pg} / \mathrm{mL}$} \\
\hline & $\mathrm{n}$ & $\%$ & $\mathrm{n}$ & $\%$ \\
\hline Children with JIA ( $\mathrm{n}=30)$ & 19 & 63 & 11 & 37 \\
\hline Healthy children $(n=30)$ & 11 & 37 & 19 & 63 \\
\hline
\end{tabular}

IL-17A enzyme-linked immunosorbent assay kit, RayBiotech, Inc., Norcross, Georgia, USA). According to the manufacturer's specifications, the minimum detectable IL-17A levels that can be determined with the use of this test is $15.63 \mathrm{pg} / \mathrm{mL}$. All the procedures were carried out on the basis of the manufacturer's instructions. The intensity of the stain reaction was determined by the ChemWell enzyme-linked immunosorbent assay reader. The absorption was measured with $450 \mathrm{~nm}$ filters through the standard curve of the serially diluted standard. Based on the standard curve, the levels of IL-17A for all 60 participants were determined.

\section{Statistical analysis}

Student's t, Mann-Whitney rank sum, Wilcoxon paired samples, Chi-square $\left(\chi^{2}\right)$, and Fisher's exact probability tests were used to assess statistical significance. Spearman's rank correlation test was used to assess correlations. A $p$ value of $<0.05$ was considered statistically significant (STATISTICA [data analysis software system] version 8.0).

\section{RESULTS}

The percentage of children with detectable and undetectable values of IL-17A in the experimental and control group is shown in Table 2. In the group of children with JIA, the percentage of detectable IL-17A levels was statistically significantly higher than in the control group $\left(\chi^{2}=4.27 ; p=0.038\right)$.

In the group of children with JIA, the lowest detected value of IL-17A was $17.18 \mathrm{pg} / \mathrm{mL}$ and the highest was $175 \mathrm{pg} / \mathrm{mL}$. In the control group, one child had IL-17A level of $24.93 \mathrm{pg} / \mathrm{mL}$, while the other 10 children with detectable IL-17A levels had borderline value of $15.63 \mathrm{pg} / \mathrm{mL}$. If the children with undetectable levels of IL-17A were eliminated, the mean values of IL-17A levels in the group of children with JIA and control group were $38.39 \mathrm{pg} / \mathrm{mL}$ and $16.47 \mathrm{pg} / \mathrm{mL}$, respectively.

Levels of IL-17A in the group of children with JIA were statistically significantly higher than in the control group (Mann-Whitney $\mathrm{U}$ test, $Z=3.384 ; p=0.001)$. Levels of IL-17A were prospectively monitored in eight children (6 with oligoarthritis and 2 with RF negative poliarthritis). In the active phase of the disease, all eight children had detectable levels of IL-17A. In the inactive phase of the disease, detectable levels of IL-17A were found in two (25\%) patients (both with oligoarthritis). Among this subgroup of children, the levels of IL-17 were significantly decreased (Wilcoxon test, $\mathrm{Z}=2.520 ; \mathrm{p}=0.012$ ) in the inactive phase of the disease.

The prevalence of detectable and undetectable IL-17A levels was also analyzed in relation to the subtypes of JIA in the group of children in the active phase of the disease (Figure 1). The majority of children with detectable IL-17A levels had oligoarthritis. Approximately two thirds of the children with oligoarthritis had IL-17A values above the threshold of detectability. If we analyzed only these 11 children with detectable IL-17A levels, the mean value was $50.69 \mathrm{pg} / \mathrm{mL}$ (range $17.18 \mathrm{pg} / \mathrm{mL}$ to $175 \mathrm{pg} / \mathrm{mL}$ ).

In more than two thirds of children with RF negative poliarthritis, serum levels of IL-17A were above $15.63 \mathrm{pg} / \mathrm{mL}$. If we analyzed serum levels of IL-17A only in this subgroup of patients, the mean concentration of IL-17A was $22.18 \mathrm{pg} / \mathrm{mL}$ (range $17.18 \mathrm{pg} / \mathrm{mL}$ to $31.25 \mathrm{pg} / \mathrm{mL}$ ). However, IL-17A was not detected in only one child with RF positive polyarthritis. 


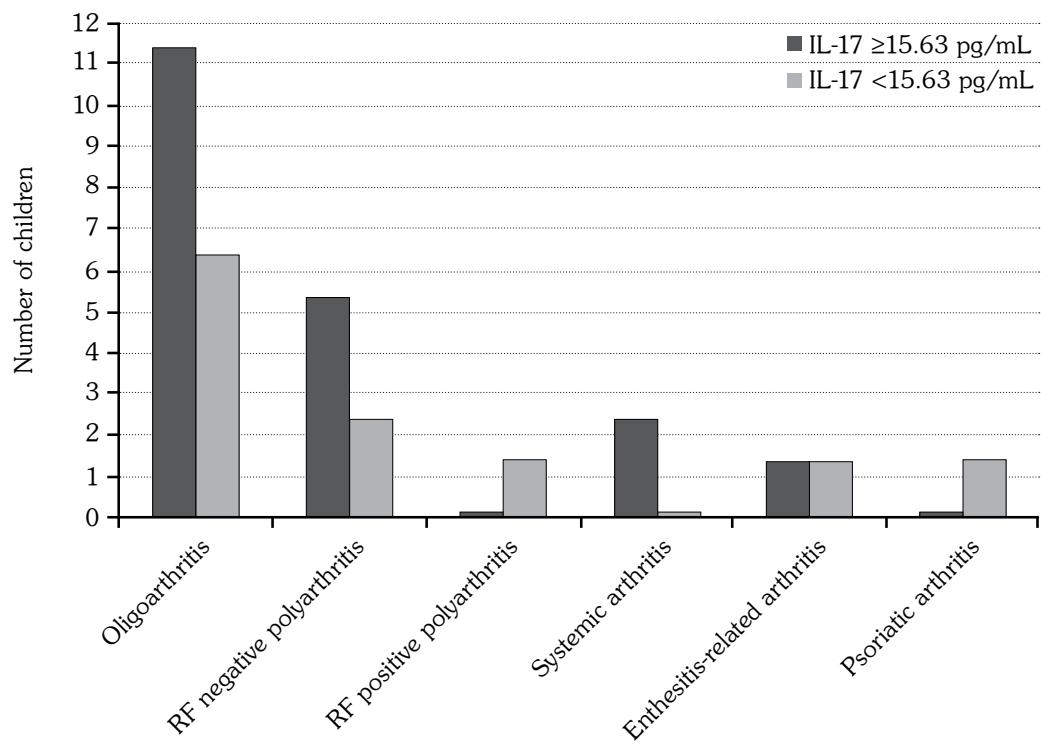

Figure 1. Prevalence of detectable and undetectable values of interleukin-17A in various subtypes of juvenile idiopathic arthritis. IL-17: Interleukin-17; RF: Rheumatoid factor.

Both patients with systemic arthritis had detectable IL-17A (mean $21.83 \mathrm{pg} / \mathrm{mL}$, range $18.73 \mathrm{pg} / \mathrm{mL}$ to $24.93 \mathrm{pg} / \mathrm{mL}$ ). One of the two children who had enthesitis-related arthritis had detectable IL-17A (17.18 pg/mL).

The analysis of significance of the difference in the prevalence of detectable IL-17A levels between oligoarthritis and polyarthritis, as the two most widespread subtypes of JIA in our sample, revealed no statistical significance (the exact probability test; $p=0.434$ ). In addition, the difference in the IL-17A levels obtained in the groups of oligoarthritis and polyarthritis was not statistically significant (Mann-Whitney $U$ test, $\mathrm{Z}=0.421 ; \mathrm{p}=0.673)$. Analysis of the frequencies of detectable levels of IL-17A in children with oligoarthritis and other forms of JIA (except polyarthritis) showed no statistically significant difference (exact probability test, $p=0.302$ ).

We analyzed clinical features and laboratory parameters in subgroups with detectable and undetectable levels of IL-17A in children with JIA. No significant differences were detected in sexes (exact probability test, $p=0.314$ ), age at onset of disease ( $\mathrm{t}$ test, $\mathrm{t}=-1.067 ; \mathrm{p}=0.294$ ), age at time of research ( $\mathrm{t}$ test, $\mathrm{t}=-0.271 ; \mathrm{p}=0.787$ ) or duration of disease ( $\mathrm{t}$ test, $\mathrm{t}=1.215 ; \mathrm{p}=0.234$ ) between the children with detectable and undetectable levels of IL-17A.
Children with JIA and detectable levels of IL-17A had significantly higher values of JADAS-27 in comparison to children with JIA and non-detectable IL-17A ( $t$ test, $p=0.029$ ).

Spreading to the cervical spine and arthritis of coxofemoral joints were only found in children with IL-17A levels above $15.63 \mathrm{pg} / \mathrm{mL}$ (3 children with affected cervical spine, seven children with arthritis of coxofemoral joints). In children with detectable levels of IL-17A, there was a statistically significant higher incidence of coxofemoral joint inflammation in comparison to the children with undetectable IL-17A levels (test of exact probability; $p=0.024)$. The difference in the incidence of arthritis with other localizations between the children with detectable and undetectable IL-17A values was not statistically significant $(p>0.05)$.

Mean values of ESR and CRP were significantly higher in the children with detectable IL-17A levels $(p<0.01)$. There was a strong positive correlation between ESR values and the levels of IL-17A (Spearman's rank correlation test, $r=0.654 ; p=0.0001)$, as well as between CRP and IL-17A values (Spearman's rank correlation test, $r=0.733 ; p=0.0004)$. The mean values of hemoglobin and thrombocytes were not significantly different between the children with detectable and undetectable IL-17A levels ( $p>0.05)$ 
Table 3. Distribution of laboratory parameters in relation to interleukin-17A levels in children with juvenile idiopathic arthritis

\begin{tabular}{|c|c|c|c|c|c|c|c|c|}
\hline \multirow[b]{2}{*}{ Level of IL-17A } & \multicolumn{2}{|c|}{$\mathrm{ESR}(\mathrm{mm} / \mathrm{h})$} & \multicolumn{2}{|c|}{$\mathrm{CRP}(\mathrm{mg} / \mathrm{L})$} & \multicolumn{2}{|c|}{$\mathrm{Hb}(\mathrm{g} / \mathrm{L})$} & \multicolumn{2}{|c|}{$\mathrm{TRC}\left(\times 10^{9} / \mathrm{L}\right)$} \\
\hline & Mean \pm SD & Range & Mean \pm SD & Range & Mean \pm SD & Range & Mean \pm SD & Range \\
\hline IL-17A >15.63 pg/mL & $57 \pm 25$ & $35-120$ & $38 \pm 29$ & $3-96$ & $117 \pm 13$ & $94-150$ & $441 \pm 136$ & $248-775$ \\
\hline IL-17A $<15.63 \mathrm{pg} / \mathrm{mL}$ & $23 \pm 14$ & $5-50$ & $6 \pm 5$ & $1-12$ & $119 \pm 12$ & $101-140$ & $392 \pm 138$ & $195-670$ \\
\hline t-test & \multicolumn{2}{|c|}{4.113} & \multicolumn{2}{|c|}{3.603} & \multicolumn{2}{|c|}{-0.329} & \multicolumn{2}{|c|}{0.933} \\
\hline$p$ & \multicolumn{2}{|c|}{0.0003} & \multicolumn{2}{|c|}{0.001} & \multicolumn{2}{|c|}{0.744} & \multicolumn{2}{|c|}{0.358} \\
\hline
\end{tabular}

(Table 3). The number of leukocytes was above $10 \times 10^{9} / \mathrm{L}$ in nine children (47\%) with detectable IL-17A levels, while leukocyte count was within the range of reference values in the group with undetectable IL-17A levels.

\section{DISCUSSION}

In this study, we analyzed the relevance of IL-17A in JIA. We found a significantly higher presence of detectable levels of IL-17A, as well as significantly higher levels of IL-17A in children in the active phase of JIA compared to healthy children. Similarly, a group of Polish authors found that serum levels of IL-17A were significantly higher in children with JIA compared to healthy children. ${ }^{27}$ In one Dutch study, increased serum levels of IL-17A were found in children with active disease and oligoarthritis or polyarthritis. ${ }^{28}$ Our study and the study of Polish authors involved mainly children with oligoarthiritis and polyarthritis, which certainly affected the results. In contrast to those results, in a recent study of a group of Italian authors, significantly elevated levels of IL-17A in children with JIA have not been detected. ${ }^{29}$

In this study, the potential role of IL-17A in the pathogenesis of JIA was also indicated by a significant drop in the level of this IL in the inactive phase of the disease in the prospectively monitored children. However, there were two children in whom the levels of IL-17A were still detectable in the inactive phase. A possible explanation is a potential residual subclinical inflammatory process, which requires further thorough monitoring of these children.

The analysis of IL-17A levels in certain subtypes of JIA confirmed that the majority of children with detectable levels of IL-17A had oligoarthritis.
Also, more than two thirds with RF negative polyarthritis had detectable levels of IL-17A. The predominance of high IL-17A levels, even higher than in our research, in children with oligoarthritis and to a lesser extent with RF negative polyarthritis in comparison to other forms of JIA was found by other authors. In two studies which assessed the levels of IL-17A in oligoarthritis, polyarthritis and systemic arthritis, the mean values of IL-17A in the serums of children with oligoarthritis in the active phase were $354 \mathrm{pg} / \mathrm{mL}$ and $450 \mathrm{pg} / \mathrm{mL}$, respectively, and the mean levels of IL-17A in children with poliarthritis in the active phase were $65 \mathrm{pg} / \mathrm{mL}$ and $100 \mathrm{pg} / \mathrm{mL}$, respectively. ${ }^{28,30}$

Latest research has partially changed the attitudes towards the pathogenic mechanisms in oligoarthritis and polyarthritis. Although the Th1 response has an important place in the pathogenesis of these forms of JIA, according to the latest findings, $\mathrm{T}$ helper (Th) 17 cells and IL-17A are singled out as a key mechanism for the induction and persistence of inflammation. ${ }^{31,32}$ This is additionally supported by the higher prevalence of Th17 cells and IL-17A in the blood and/or synovial fluid of children with oligoarthritis and polyarthritis. ${ }^{22,28,30}$ The aggrecan peptides from the extracellular matrix belong to the most significant autoantigens that activate Th17 cells and induce the production of IL-17A in these children. ${ }^{33}$ In addition, proinflammatory cytokines, such as IL- 6 or IL- $1 \beta$ in the microenvironment inside the joints of children with oligoarthritis and polyarthritis favor the differentiation of naive CD4+ T cells (Th0 lymphocytes) towards Th17 cells, and this leads to the increased production of IL-17A. ${ }^{31}$

In our study, two children had systemic arthritis in the active phase, and both had detectable IL-17A levels, but this number is insufficient for 
drawing valid conclusions. One of the crucial mechanisms involved in development of systemic arthritis is the uncontrolled activation of the innate immune response, which results in the release of proinflammatory cytokines such as IL-1, IL-6,

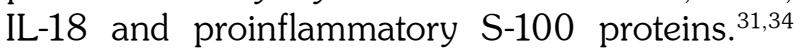
However, a study by British authors confirmed a significant presence of Th17 cells which secrete IL-17A in children with systemic arthritis. ${ }^{35}$ In contrast, in two studies that analyzed the levels of IL-17A in children with oligoarthritis, polyarthritis and systemic arthritis, IL-17A levels were not elevated in children with systemic arthritis. ${ }^{28,30}$

In our study, the difference in the incidence of detectable levels of IL-17A and the difference in IL-17A values between oligoarthritis and polyarthritis, the two predominant forms of JIA in the group studied, were not significant. Results of other authors indicate significantly higher IL-17A levels in children with oligoarthritis in comparison to other forms of JIA and significantly higher IL-17A levels in children with polyarthritis in comparison to systemic arthritis. ${ }^{28,30}$ The results of the studies mentioned confirm the role of IL-17A in the pathogenesis of oligoarthritis and polyarthritis. The inconsistency of our results with the results of other authors point to the need for further research on a larger sample.

The question that arises whether patients with detectable and undetectable IL-17A levels in the active phase of the disease could be differentiated on the basis of certain clinical and laboratory characteristics. The levels of IL-17A do not correlate with sex, age at onset of disease, age at time of research or duration of disease. Accurate appraisal of disease activity has a substantial role in the assessment of disease severity and prediction of disease outcome. ${ }^{24}$ In our study, values of JADAS-27 were significantly higher in children with detectable IL-17A levels. In the available literature, there are no data on the relationship of IL-17A and values of JADAS-27 in children with JIA. Inflammation of the spine and coxofemoral joints is an indicator of more serious forms of the disease. ${ }^{19,36,37}$ In our study, spreading to the cervical spine and coxofemoral joints arthritis were only found in children with detectable IL-17A values in the active phase of JIA. Therefore, children with detectable IL-17A levels should be closely monitored, and such results imply that, on the basis of IL-17A values in the initial phase of JIA, it may be possible to select patients at a higher risk from a worse functional outcome.

The mean ESR values were significantly higher in children with detectable IL-17A levels in comparison to those with undetectable IL-17A values. In a study by Indian authors, which assessed the correlation between ESR and IL-17A levels in the synovial fluid, no significant difference was found. ${ }^{22}$ The difference in the results obtained may be the consequence of different methodologies of IL-17A identification and in the incomparability of the groups researched, since in our study, the prevalent forms were oligoarthritis and polyarthritis, while the study by the Indian authors dealt with enthesitis-related arthritis.

$\mathrm{C}$-reactive protein is a more sensitive indicator of inflammation, because it reflects the real current inflammation level and spread of the disease. ${ }^{38}$ In our study, mean values of CRP were significantly higher in children with detectable levels of IL-17A, in comparison with the group of children with undetectable values of IL-17A. The synthesis of CRP is stimulated by IL- 1 and IL-6. ${ }^{38}$ The biological activity of IL-17A is realized through various mechanisms, one of them being the stimulation of production of various cytokines, such as IL-1, IL- 6 and others, ${ }^{39}$ which may be an explanation of the positive correlation of IL-17A levels and CRP. However, the results of some studies indicate that IL-17A directly stimulates hepatocytes to produce CRP, independently from IL-1 and IL-6. ${ }^{39-41}$ Strong positive correlation between values of IL-17A levels and the values of ESR and CRP in the active phase of JIA, which was identified in our study, indirectly points to the possible association of IL-17A with the severity and extensiveness of inflammation.

The increased leukocyte and thrombocyte counts and decreased hemoglobin values are other reliable markers of inflammation and degree of disease activity. In this study, an elevated leukocyte number was found in $47 \%$ of children in the group with detectable IL-17A values, and only one of them had systemic arthritis. To our knowledge, there are no data in the literature on the relationship of IL-17A and leukocyte number in children with JIA. However, in the patients with rheumatic arthritis, a significant correlation 
was found between IL-17A levels in the synovial fluid and the number of leukocytes. ${ }^{42}$ In addition, the number of Th17 cells significantly correlated with the number of leukocytes in the synovial fluid. ${ }^{43}$

In our study, no significant difference was found in the mean values of hemoglobin between children with detectable and undetectable IL-17A levels. Also, no similar data on IL-17A and hemoglobin values in children with JIA were detected in the literature. However, analysis of the relationship of IL-17A and hemoglobin levels showed no significant correlation in patients with rheumatoid arthritis. ${ }^{42}$

No significant difference was found in our study in the mean thrombocyte count in children with detectable and undetectable IL-17A levels. Taking in consideration that the emergence of thrombocytosis in inflammation, including JIA, is primarily the consequence of IL- 1 , tumor necrosis factor alpha and IL- $6,{ }^{44,45}$ while IL-17A stimulates the production and displays the synergetic effects with these cytokines, ${ }^{39}$ the correlation of IL-17A levels and thrombocyte count could be expected, although it has not been proved in our study.

The main limitation of this study is a relatively small number of patients included. Evaluation of the accuracy of the results requires further confirmation in clinical practice and on a larger sample of patients, what may be the subject of some future studies.

In conclusion, precise defining of the role of IL-17A in JIA may contribute to understanding immune mechanisms which are not entirely explained, as well as the functional outcome, regarding the destructive potential of this IL. This study gives a small contribution to determining the significance of IL-17A in JIA. Increased levels of IL-17A in active phase of JIA, as well as significant decrease in serum IL-17A levels in prospectively followed group of patients in inactive phase of JIA, pinpoint to possible role of IL-17A in the pathogenesis of JIA. In our study, we found significantly higher values of JADAS-27 in group of children with detectable IL-17A levels. Also, arthritis of coxofemoral joints was only found in children with detectable IL-17A values. These facts are important because they open a window of possibility for early detection of children that have higher risk for worse functional outcome if IL-17A would be determined in early phases of JIA.

\section{Declaration of conflicting interests}

The authors declared no conflicts of interest with respect to the authorship and/or publication of this article.

\section{Funding}

The authors received no financial support for the research and/or authorship of this article.

\section{REFERENCES}

1. Leipe J, Grunke M, Dechant C, Reindl C, Kerzendorf $\mathrm{U}$, Schulze-Koops $\mathrm{H}$, et al. Role of Th17 cells in human autoimmune arthritis. Arthritis Rheum 2010;62:2876-85.

2. Astry B, Venkatesha $\mathrm{SH}$, Moudgil KD. Involvement of the IL-23/IL-17 axis and the Th17/Treg balance in the pathogenesis and control of autoimmune arthritis. Cytokine 2015;74:54-61.

3. Roeleveld DM, Koenders MI. The role of the Th17 cytokines IL-17 and IL-22 in Rheumatoid Arthritis pathogenesis and developments in cytokine immunotherapy. Cytokine 2015;74:101-7.

4. Benedetti G, Miossec P. Interleukin 17 contributes to the chronicity of inflammatory diseases such as rheumatoid arthritis. Eur J Immunol 2014;44:339-47.

5. Ryu S, Lee JH, Kim SI. IL-17 increased the production of vascular endothelial growth factor in rheumatoid arthritis synoviocytes. Clin Rheumatol 2006;25:16-20.

6. Toh ML, Gonzales G, Koenders MI, Tournadre A, Boyle D, Lubberts E, et al. Role of interleukin 17 in arthritis chronicity through survival of synoviocytes via regulation of synoviolin expression. PLoS One 2010;5:13416.

7. Li X, Yuan FL, Lu WG, Zhao YQ, Li CW, Li JP, et al. The role of interleukin-17 in mediating joint destruction in rheumatoid arthritis. Biochem Biophys Res Commun 2010;397:131-5.

8. Wang M, Tian T, Yu S, He N, Ma D. Th17 and Treg cells in bone related diseases. Clin Dev Immunol 2013;2013:203705.

9. Cascão R, Moura RA, Perpétuo I, Canhão H, VieiraSousa E, Mourão AF, et al. Identification of a cytokine network sustaining neutrophil and Th17 activation in untreated early rheumatoid arthritis. Arthritis Res Ther 2010;12:196.

10. Lubberts E, Koenders MI, van den Berg WB. The role of T-cell interleukin-17 in conducting destructive arthritis: lessons from animal models. Arthritis Res Ther 2005;7:29-37.

11. Kleyer A, Schett G. Arthritis and bone loss: a hen and egg story. Curr Opin Rheumatol 2014;26:80-4. 
12. Adamopoulos IE, Chao CC, Geissler R, Laface $\mathrm{D}$, Blumenschein $\mathrm{W}$, Iwakura $\mathrm{Y}$, et al. Interleukin17A upregulates receptor activator of NF-kappaB on osteoclast precursors. Arthritis Res Ther 2010;12:29.

13. Zupan J, Jeras M, Marc J. Osteoimmunology and the influence of pro-inflammatory cytokines on osteoclasts. Biochem Med (Zagreb) 2013;23:43-63.

14. Al-Saadanya HM, Husseina MS, Gaberb RA, Zaytounc HA. TH-17 cells and serum IL-17 in rheumatoid arthritis patients: Correlation with disease activity and severity. Egypt Rheumatol 2016;38:1-7.

15. Lazic E, Jelusic M, Grcevic D, Marusic A, Kovacic N. Osteoblastogenesis from synovial fluid-derived cells is related the type and severity of juvenile idiopathic arthritis. Arthritis Res Ther 2012;14:139.

16. Paulissen SMJ, Van Hamburg JP, Davelaar N, Asmawidjaja PS, Hazes JM, Lubberts E. Synovial fibroblasts directly induce Th17 pathogenicity via the cyclooxygenase/prostaglandin E2 pathway, independent of IL-23. J Immunol 2013;191:1364-72.

17. Southwood TR. Classifying childhood arthritis. Ann Rheum Dis 1997;56:79-81.

18. Petty RE, Southwood TR, Manners P, Baum J, Glass $\mathrm{DN}$, Goldenberg $\mathrm{J}$, et al. International League of Associations for Rheumatology classification of juvenile idiopathic arthritis: second revision, Edmonton, 2001. J Rheumatol 2004;31:390-2.

19. Cassidy TJ, Petty ER. Chronic arthritis in childhood. In: Cassidy TJ, Petty ER, editors. Textbook of Pediatric Rheumatology. 5th ed. Philadelphia: W.B. Saunders Company; 2005. p. 206-60.

20. Shimizu M, Nakagishi Y, Yachie A. Distinct subsets of patients with systemic juvenile idiopathic arthritis based on their cytokine profiles. Cytokine 2013;61:345-8.

21. Mahendra A, Misra R, Aggarwal A. Th1 and Th17 Predominance in the Enthesitis-related Arthritis Form of Juvenile Idiopathic Arthritis. J Rheumatol 2009;36:1730-6.

22. Agarwal S, Misra R, Aggarwal A. Interleukin 17 levels are increased in juvenile idiopathic arthritis synovial fluid and induce synovial fibroblasts to produce proinflammatory cytokines and matrix metalloproteinases. J Rheumatol 2008;35:515-9.

23. Nistala K, Wedderburn LR. Th17 and regulatory $T$ cells: rebalancing pro- and anti-inflammatory forces in autoimmune arthritis. Rheumatology (Oxford) 2009;48:602-6.

24. Consolaro A, Ruperto N, Bazso A, Pistorio A, Magni-Manzoni S, Filocamo G, et al. Development and validation of a composite disease activity score for juvenile idiopathic arthritis.Arthritis Rheum 2009;61:658-66

25. Wallace CA, Giannini EH, Huang B, Itert L, Ruperto N. American College of Rheumatology provisional criteria for defining clinical inactive disease in select categories of juvenile idiopathic arthritis. Arthritis
Care Res (Hoboken) 2011;63:929-36.

26. Consolaro A, Negro G, Lanni S, Solari N, Martini A, Ravelli A. Toward a treat-to-target approach in the management of juvenile idiopathic arthritis. Clin Exp Rheumatol 2012;30:157-62.

27. Szymanska-Kaluza J, Cebula-Obrzut B, Smolewski P, Stanczyk J, Smolewska E. Imbalance of Th17 and T-regulatory cells in peripheral blood and synovial fluid in treatment naïve children with juvenile idiopathic arthritis. Cent Eur J Immunol 2014;39:71-6.

28. de Jager W, Hoppenreijs EP, Wulffraat NM, Wedderburn LR, Kuis W, Prakken BJ. Blood and synovial fluid cytokine signatures in patients with juvenile idiopathic arthritis: a cross-sectional study. Ann Rheum Dis 2007;66:589-98.

29. Cosmi L, Cimaz R, Maggi L, Santarlasci V, Capone $\mathrm{M}$, Borriello $\mathrm{F}$, et al. Evidence of the transient nature of the Th17 phenotype of CD4+CD161+ T cells in the synovial fluid of patients with juvenile idiopathic arthritis. Arthritis Rheum 2011;63:2504-15.

30. van den Ham HJ, de Jager W, Bijlsma JW, Prakken BJ, de Boer RJ. Differential cytokine profiles in juvenile idiopathic arthritis subtypes revealed by cluster analysis. Rheumatology (Oxford) 2009;48:899-905.

31. Lin YT, Wang CT, Gershwin ME, Chiang BL. The pathogenesis of oligoarticular/polyarticular vs systemic juvenile idiopathic arthritis. Autoimmun Rev 2011;10:482-9.

32. Cimaz R, Moretti D, Pagnini I, Marino A, Cantarini L, Simonini G. What do cytokine profiles tell us about subsets of juvenile idiopathic arthritis? Curr Rheumatol Rep 2012;14:150-4.

33. Kamphuis S, Hrafnkelsdóttir K, Klein MR, de Jager W, Haverkamp MH, van Bilsen $\mathrm{JH}$, et al. Novel selfepitopes derived from aggrecan, fibrillin, and matrix metalloproteinase- 3 drive distinct autoreactive T-cell responses in juvenile idiopathic arthritis and in health. Arthritis Res Ther 2006;8:178.

34. Prakken B, Albani S, Martini A. Juvenile idiopathic arthritis. Lancet 2011;377:2138-49.

35. Omoyinmi E, Hamaoui R, Pesenacker A, Nistala $\mathrm{K}$, Moncrieffe H, Ursu S, et al. Th1 and Th17 cell subpopulations are enriched in the peripheral blood of patients with systemic juvenile idiopathic arthritis. Rheumatology (Oxford) 2012;51:1881-6.

36. Rostom S, Amine B, Bensabbah R, Abouqal R, HajjajHassouni N. Hip involvement in juvenile idiopathic arthritis. Clin Rheumatol 2008;27:791-4.

37. Elhai M, Wipff J, Bazeli R, Freire V, Feydy A, Drapé $\mathrm{JL}$, et al. Radiological cervical spine involvement in young adults with polyarticular juvenile idiopathic arthritis. Rheumatology (Oxford) 2013;52:267-75.

38. Agarwal M, Sawhney S. Laboratory tests in pediatric rheumatology. Indian J Pediatr 2010;77:1011-6.

39. Xu S, Cao X. Interleukin-17 and its expanding biological functions. Cell Mol Immunol 2010;7:164-74.

40. Patel DN, King CA, Bailey SR, Holt JW, Venkatachalam $\mathrm{K}$, Agrawal A, et al. Interleukin-17 stimulates 
C-reactive protein expression in hepatocytes and smooth muscle cells via p38 MAPK and ERK1/2dependent NF-kappaB and C/EBPbeta activation. J Biol Chem 2007;282:27229-38.

41. Eklund CM. Proinflammatory cytokines in CRP baseline regulation. Adv Clin Chem 2009;48:111-36.

42. Metawi SA, Abbas D, Kamal MM, Ibrahim MK. Serum and synovial fluid levels of interleukin-17 in correlation with disease activity in patients with RA. Clin Rheumatol 2011;30:1201-7.

43. Zizzo G, De Santis M, Bosello SL, Fedele AL,
Peluso G, Gremese E, et al. Synovial fluid-derived T helper 17 cells correlate with inflammatory activity in arthritis, irrespectively of diagnosis. Clin Immunol 2011;138:107-16.

44. Bleeker JS, Hogan WJ. Thrombocytosis: diagnostic evaluation, thrombotic risk stratification, and risk-based management strategies. Thrombosis 2011;2011:536062.

45. Cimaz R, Von Scheven A, Hofer M. Systemic-onset juvenile idiopathic arthritis: the changing life of a rare disease. Swiss Med Wkly 2012;142:13582. 